\title{
Computationally Efficient HQP-based Whole-body Control Exploiting the Operational-space Formulation
}

\author{
Yisoo Lee ${ }^{1}$, Junewhee $\mathrm{Ahn}^{2}$, Jinoh Lee ${ }^{3}$, and Jaeheung Park ${ }^{2}$
}

\begin{abstract}
This paper proposes a novel and practical approach to enhance the computational efficiency of the hierarchical quadratic programming (HQP)-based whole-body control. The HQP method is known to offer control solutions satisfying strict priority with various constraints for multipletasks execution. However, it inherently comes at the price of high computation time to solve QP optimization problems in each hierarchical level which limits practicability in a realtime control system with fast sampling time. To mitigate this issue, we propose that the operational space formulation is incorporated into the HQP method, where the decision variables are intuitively defined at the task level and possess smaller dimensions. Indeed, it serves faster whole-body control solution for multiple tasks under equality and inequality constraints yet strictly fulfilling the task priority. The performance of the proposed method is experimentally verified on the actual floatingbased humanoid, named TOCABI with 33 degrees-of-freedom. In addition, computation time is analyzed by comparison with conventional HQP and other advanced implementation forms.
\end{abstract}

\section{INTRODUCTION}

Whole-body control (WBC) is a complex problem demanding dynamic and stable motion of a floating base robot with many degrees of freedom (DoFs), e.g., balancing and locomotion of humanoid robots. In such practical WBC scenarios, it is mandatory to systematically perform multiple tasks while maintaining stable contacts with environment, where an optimal control output must be found among the infinite number of solutions with consideration of various equality and inequality constraints. Besides, the WBC algorithm is obliged to complete the computation in a real-time control loop with high sampling frequency to guarantee the actual performance.

A breakthrough to solve the complicated WBC problem with equality and inequality constraints comes with a Quadratic programming (QP) optimization [1]. The QPbased WBC formulation offers straightforward and easy-toimplement structure, where robot dynamics and kinematics are set as the hard constraints while the inequality constraints

This work was supported by the National Research Foundation (NRF) of Korea funded by the Ministry of Education and the Korea government (MSIT) (No. 2019R1A6A3A03033473, No. 2021R1A2C3005914). This work was also supported by the Korea Institute of Science and Technology Institutional Programs under grant number 2E31063. (Yisoo Lee and Junewhee Ahn are co-first authors.) (Corresponding Author: Jinoh Lee.)

${ }^{1} \mathrm{Y}$. Lee is with Center for Intelligent and Interactive Robotics, Korea Institute of Science and Technology (KIST), Seoul 02792, South Korea.

${ }^{2}$ J. Ahn and J. Park are with the Graduate School of Convergence Science and Technology, Seoul National University (SNU), Gwanak-ro 1, Gwanakgu, Seoul, Republic of Korea.

${ }^{3} \mathrm{~J}$. Lee is with the Institute of Robotics and Mechatronics, German Aerospace Center (DLR), 82234, Weßling, Germany. jinoh.leeddlr.de are satisfied for stable contacts, physical limits of joints, and other practical conditions [2]-[4]. However, the QPbased methods manage multiple tasks by assigning different weightings in the cost function, which results in soft task hierarchy, i.e., the higher priority task performance cannot be guaranteed due to the impact from the lower priority tasks when any tasks are in conflict.

On the other hand, the operational space formulation (OSF)-based WBC method captures the capability to control multiple tasks with strict hierarchy [5]. With the null-space projection approach, the higher priority task is respected while the lower priority task under conflict is sacrificed. The effectiveness of the OSF-based whole-body controller is also verified through real robot experiments [6]-[8]. Nevertheless, there is a crucial limitation to handle general inequality constraints. While a task transition method [9] may allow the OSF consider certain inequality constraints, e.g., joint limits, the method requires to add many tasks and complex transition algorithms when there are multiple constraints.

To cope with both strict task hierarchy and inequality constraints, the QP-based method is extended as the hierarchical QP (HQP) formulation [10]. This generic method inherits all the advantages of the QP-based method while generating a strict task hierarchy as the OSF-based one. In return for the strict hierarchy in HQP, however, it requires a relatively long computation time since it has to solve multiple QPs as many as the number of prioritized task. The computational cost issue becomes crucial especially when solving inverse dynamics problem for real-time control of many DoFs robot in high control frequency, e.g., humanoids with $1 \mathrm{kHz}$ sampling period. Therefore, to reduce computation cost of the HQP, several methods have been proposed: for example, authors in [11] suggest to decrease the number of equality and inequality constraints; [12] enables handling of inequality constraints on any priority level; the method in [13], [14] reduces the size of decision variablet by decoupling the robot dynamics equation; and the authors in [15] propose a variable reducing elimination method based on the choice of null-space basis. Despite these efforts, the challenge still comes with its inherently high computation for the HQP to implement sufficiently high numbers of hierarchical wholebody tasks for high DoFs robots.

Accordingly, this paper aspires to develope a fast HQPbased WBC by exploiting the OSF which plays an effective role to reduce the dimension of the decision variables for each QP. In addition, the proposed HQP inherits the capability to explicitly control the tasks in force level from the nature of the OSF, unlike the conventional HQP only deals 
with the acceleration-level tasks. Real robot experiments are conducted to evaluate the efficacy of the proposed method with 33-DoFs humanoid robot TOCABI performing five hierarchical whole-body tasks. For deeper investigation of computational efficiency, the compuation time of the proposed method is numerically compared with that of conventional HQP as well as its advanced implementation schemes known to improve the speed of HQP.

\section{BACKGROUND ON THE HQP-BASED WBC}

In this section, we introduce concept of the conventional HQP-based WBC method for floating base robot. Generally, the Jacobian matrix and robot dynamics are employed for the inverse dynamics solution. Thus, we first explain them and then introduce the concept of the HQP.

The velocity of operational space $\mathbf{x}$ of the robot with $n$ DoFs is described as $\dot{\mathbf{x}}=\mathbf{J} \dot{\mathbf{q}}$, where $\mathbf{J}$ is the Jacobian matrix and $\mathbf{q} \in \mathbb{R}^{n}$ is the robot configuration vector. By differentiating the velocity equation, the acceleration $\ddot{x}$ can be given as $\ddot{\mathbf{x}}=\mathbf{J} \ddot{\mathbf{q}}+\dot{\mathbf{J}} \dot{\mathbf{q}}$. Rigid body dynamics of the floating base robot which has $n$ DoFs, with $k=n-6$ joints, and $c$ DoFs contacts can be expressed as

$$
\mathbf{A} \ddot{\mathbf{q}}+\mathbf{h}+\mathbf{J}_{c}^{T} \mathbf{F}_{c}=\mathbf{S}^{T} \mathbf{\Gamma},
$$

where $\mathbf{A} \in \mathbb{R}^{n \times n}$ is the inertia matrix, $\mathbf{h} \in \mathbb{R}^{n}$ is the generalized force vector including the Coriolis/centrifugal and gravity effects, $\mathbf{J}_{c} \in \mathbb{R}^{c \times n}$ is the contact Jacobian matrix where the relation $\dot{\mathbf{x}}_{c}=\mathbf{J}_{c} \dot{\mathbf{q}}$ holds, $\mathbf{x}_{c} \in \mathbb{R}^{c}$ is the contact space vector describing positions and orientations of the contact links, $\mathbf{F}_{c} \in \mathbb{R}^{c}$ is the contact wrench vector, $\mathbf{S} \in \mathbb{R}^{k \times n}$ is a selection matrix to exclude un-actuated joints, and $\Gamma \in \mathbb{R}^{k}$ is the actual joint torque vector.

For a single task of the floating base robot, a QP optimization problem then can be formulated as follows:

$$
\begin{gathered}
\min _{\ddot{\mathbf{q}}, \boldsymbol{\Gamma}, \mathbf{F}_{c}, \mathbf{w}_{1}}\left\|\mathbf{w}_{1}\right\|^{2}, \\
\text { subject to }\left\{\begin{array}{l}
\mathbf{A} \ddot{\mathbf{q}}+\mathbf{h}+\mathbf{J}_{c}^{T} \mathbf{F}_{c}=\mathbf{S}^{T} \boldsymbol{\Gamma}, \\
\mathbf{J}_{1} \ddot{\mathbf{q}}+\dot{\mathbf{J}}_{1} \dot{\mathbf{q}}=\ddot{\mathbf{x}}_{1}^{d}+\mathbf{w}_{1}, \\
\mathbf{J}_{c} \ddot{\mathbf{q}}+\dot{\mathbf{J}}_{c} \dot{\mathbf{q}}=\mathbf{0},
\end{array}\right.
\end{gathered}
$$

and additional inequality constraints for stationary contacts, i.e., friction cone and center of pressure $(\mathrm{CoP})$, associated with $\mathbf{F}_{c}$, and joint limits associated with $\ddot{\mathbf{q}}$ and $\boldsymbol{\Gamma}$. Here, $\mathbf{w}_{1}$ is a slack variable vector, $\ddot{\mathbf{x}}_{1}^{d}$ is the desired acceleration vector at the operational space, and the number in the subscript of $\mathbf{w}_{\bullet}, \ddot{\mathbf{x}}_{\bullet}^{d}$ and $\mathbf{J}_{\bullet}$ denote associated task priority.

Rigid and stationary contacts can be guaranteed by considering the robot dynamics (3) with contact condition (5) and inequality constraints regarding the friction cone and CoP. Note that (5) has the physical meaning as $\ddot{\mathbf{x}}_{c}=\mathbf{0}$ which is required for stationary contacts. As a result, with (4), $\ddot{\mathbf{x}}_{1}^{d}+\mathbf{w}_{1}$ will be generated at the operational space while satisfying all the constraints when the obtained optimal solution $\Gamma$ is commanded to the joint torque controller of robot.

When there is a second priority task, one more QP has to be solved in addition to that of the first priority task. The
QP optimization for the second priority can be formulated as follows:

$$
\begin{aligned}
& \min _{\ddot{\mathbf{q}}, \boldsymbol{\Gamma}, \mathbf{F}_{c}, \mathbf{w}_{2}}\left\|\mathbf{w}_{2}\right\|^{2}, \\
& \text { subject to }\left\{\begin{array}{l}
\mathbf{A} \ddot{\mathbf{q}}+\mathbf{h}+\mathbf{J}_{c}^{T} \mathbf{F}_{c}=\mathbf{S}^{T} \boldsymbol{\Gamma}, \\
\mathbf{J}_{1} \ddot{\mathbf{q}}+\dot{\mathbf{J}}_{1} \dot{\mathbf{q}}=\ddot{\mathbf{x}}_{1}^{d}+\mathbf{w}_{1}^{*}, \\
\mathbf{J}_{2} \ddot{\mathbf{q}}+\dot{\mathbf{J}}_{2} \dot{\mathbf{q}}=\ddot{\mathbf{x}}_{2}^{d}+\mathbf{w}_{2}, \\
\mathbf{J}_{c} \ddot{\mathbf{q}}+\dot{\mathbf{J}}_{c} \dot{\mathbf{q}}=\mathbf{0},
\end{array}\right.
\end{aligned}
$$

and additional inequality constraints for stationary contacts and joint limits. In the above $\mathrm{QP}$, slack variable $\mathbf{w}_{2}$ relaxes the second priority task while strictly satisfying the equality constraint (8). The constraint (8) is set to guarantee a strict task hierarchy in which the lower priority task does not affect higher priority. In this constraint, $\mathbf{w}_{1}^{*}$ is a constant vector that is optimized in (2). Thus, the optimal solution of (6) does not affect the result from the first QP (2). After solving the secondary QP, $\Gamma$ from (6) generates optimized task space acceleration $\ddot{\mathbf{x}}_{1}^{d}+\mathbf{w}_{1}$ and $\ddot{\mathbf{x}}_{2}^{d}+\mathbf{w}_{2}$ for the first and second priority task, respectively.

A control solution for more hierarchical tasks can be obtained by extending the above cascade QPs as many as the number of task hierarchy. The generalized formulation for $l$-th prioritized task can be expressed as follows:

$$
\begin{aligned}
& \min _{\ddot{\mathbf{q}}, \boldsymbol{\Gamma}, \mathbf{F}_{c}, \mathbf{w}_{l}}\left\|\mathbf{w}_{l}\right\|^{2}, \\
& \text { subject to }\left\{\begin{array}{l}
\mathbf{A} \ddot{\mathbf{q}}+\mathbf{h}+\mathbf{J}_{c}^{T} \mathbf{F}_{c}=\mathbf{S}^{T} \boldsymbol{\Gamma}, \\
\mathbf{J}_{i} \ddot{\mathbf{q}}+\dot{\mathbf{J}}_{i} \dot{\mathbf{q}}=\ddot{\mathbf{x}}_{i}^{d}+\mathbf{w}_{i}^{*}, \forall i \in 1, \cdots, l-1 \\
\mathbf{J}_{l} \ddot{\mathbf{q}}+\dot{\mathbf{J}}_{l} \dot{\mathbf{q}}=\ddot{\mathbf{x}}_{l}^{d}+\mathbf{w}_{l}, \\
\mathbf{J}_{c} \ddot{\mathbf{q}}+\dot{\mathbf{J}}_{c} \dot{\mathbf{q}}=\mathbf{0} .
\end{array}\right.
\end{aligned}
$$

When there is redundancy after solving the above QPs in order from $l=1$ to $m$, where $m$ is the number of hierarchical tasks, an additional QP can be solved for regularization to make the HQP full-rank. For example, joint torque or contact wrench can be minimized in the additional QP. Then, the optimal solution of the last QP can be provided for the wholebody controller.

\section{A FAST HQP-BASED WBC EXPLOITING THE OSF}

In this section, we propose to apply the contact consistent OSF to the HQP formulation to enhance the computational efficiency. Employing the OSF instead of (3), (13) and (5) reduces the number of the decision variable and as a consequence, the computation time of the new HQP-based WBC can be greatly decreased.

\section{A. Proposed Method}

In the conventional contact consistent OSF [5], the control torque for $m$ hierarchical tasks can be described as follows:

$$
\boldsymbol{\Gamma}=\widetilde{\mathbf{J}}_{1}^{T} \boldsymbol{\Lambda}_{1} \ddot{\mathbf{x}}_{1}^{d}+\sum_{i=2}^{m}\left\{\left(\prod_{j=1}^{i-1} \widetilde{\mathbf{N}}_{j}^{T}\right) \widetilde{\mathbf{J}}_{i}^{T} \boldsymbol{\Lambda}_{i} \ddot{\mathbf{x}}_{i}^{d}\right\}+\mathbf{Y}_{c} \mathbf{F}_{c}^{r}+\boldsymbol{\Gamma}_{h}
$$

where $\widetilde{\mathbf{J}}_{\bullet}^{T}=\overline{\overline{\mathbf{J}}_{\bullet}^{T} \mathbf{S}^{T}}$ is the matrix that expresses torque-force relation with consideration of under-actuation for $\bullet$-th task, $\mp$ is dynamically consistent inverse of $\star, \Lambda_{\bullet}$ is the inertia 
matrix at the operational space, $\widetilde{\mathbf{N}}_{\bullet}^{T}=\mathbf{I}-\widetilde{\mathbf{J}}_{\bullet}^{T} \widetilde{\mathbf{J}}_{\bullet}^{T}$ is the nullspace projection matrix, $\mathbf{Y}_{c} \in \mathbb{R}^{k \times(c-6)}$ is a mapping matrix proposed in [16] for calculating the torque that can modify contact wrench without affecting the operational space tasks, $\mathbf{F}_{c}^{r} \in \mathbb{R}^{c-6}$ is the contact wrench distribution vector, and $\boldsymbol{\Gamma}_{h}$ is a torque vector for compensate $\mathbf{h}$.

Accordingly, when the model is the same as the real robot and $\boldsymbol{\Gamma}_{h}$ perfectly compensates for gravity and Coriolis/centrifugal forces, desired accelerations of hierarchical tasks can be generated by the first two terms on the righthand side of (16), and the contact wrench can be modified by $\mathbf{F}_{c}^{r}$ without affecting the tasks through the third term. In the contact consistent OSF, contact wrench $\mathbf{F}_{c}$ can be calculated through the contact constrained dynamics equation as follows:

$$
\mathbf{F}_{c}=\overline{\mathbf{J}}_{c}^{T} \mathbf{S}^{T} \boldsymbol{\Gamma}+\mathbf{h}_{c},
$$

where $\mathbf{h}_{c}$ is the generalized force vector in which $\mathbf{h}$ is projected on the contact space. Based on the above equations (16) and (17), the operational space-based HQP is developed in this study.

For the first prioritized task, formulation of the operational space-based optimization can be expressed as follows:

subject to $\left\{\begin{array}{l}\boldsymbol{\Gamma}=\widetilde{\mathbf{J}}_{1}^{T} \boldsymbol{\Lambda}_{1}\left(\ddot{\mathbf{x}}_{1}^{d}+\mathbf{w}_{1}\right)+\mathbf{Y}_{c} \mathbf{F}_{c}^{r}+\boldsymbol{\Gamma}_{h}, \\ \boldsymbol{\Gamma} \in\left[\boldsymbol{\Gamma}^{\min }, \boldsymbol{\Gamma}^{\max }\right], \\ \ddot{\mathbf{x}}_{1}^{d}+\mathbf{w}_{1} \in\left[\ddot{\mathbf{x}}^{\min }, \ddot{\mathbf{x}}^{\max }\right], \\ \mathbf{K F}_{c} \in\left[\mathbf{f}_{c}^{\min }, \mathbf{f}_{c}^{\max }\right],\end{array}\right.$

where (19) expresses the torque-task relationship from the contact-consistent OSF (16) for a single task; (21) the joint constraint; (22) the task constraint, where the vector $\ddot{\mathrm{x}}^{\text {min }}$ and $\ddot{\mathrm{x}}^{\mathrm{max}}$ are lower and upper limits of the task acceleration, respectively; and (22) the contact wrench constraints, where $\mathbf{K}$ is a mapping matrix that transforms the contact wrench into friction cones and $\mathrm{CoP}$ constraints [17], and the vectors $\mathbf{f}_{c}^{\min }$ and $\mathbf{f}_{c}^{\max }$ describe lower and upper limits of the friction cone and CoP boundaries.

Among the above constraints, the torque-task relationship (19) and the contact wrench (22) constraints are essential, while inequalities (21) and (22) are optional. Thus, those can be configured when joint limits or task limits exist.

The above constraints (19), (21), and (22) can be reformulated into three inequalities for QP formulation. By substituting (19) into (21), joint torque limit can be given as

$$
\widetilde{\mathbf{J}}_{1}^{T} \boldsymbol{\Lambda}_{1}\left(\ddot{\mathbf{x}}_{1}^{d}+\mathbf{w}_{1}\right)+\mathbf{Y}_{c} \mathbf{F}_{c}^{r}+\boldsymbol{\Gamma}_{h} \in\left[\boldsymbol{\Gamma}^{\min }, \boldsymbol{\Gamma}^{\max }\right] .
$$

Substituting (17) and (19) into (22) yields the contact wrench constraint as follows:

$$
\begin{aligned}
\mathbf{K} \mathbf{F}_{c} & =\mathbf{K}\left(\overline{\mathbf{J}}_{c}^{T} \mathbf{S}^{T} \boldsymbol{\Gamma}+\mathbf{h}_{c}\right) \\
& =\mathbf{K} \overline{\mathbf{J}}_{c}^{T} \mathbf{S}^{T} \widetilde{\mathbf{J}}_{1}^{T} \boldsymbol{\Lambda}_{1}\left(\ddot{\mathbf{x}}_{1}^{d}+\mathbf{w}_{1}\right) \\
& +\mathbf{K} \overline{\mathbf{J}}_{c}^{T} \mathbf{S}^{T}\left(\mathbf{Y}_{c} \mathbf{F}_{c}^{r}+\boldsymbol{\Gamma}_{h}\right)+\mathbf{K} \mathbf{h}_{c} \in\left[\mathbf{f}_{c}^{\min }, \mathbf{f}_{c}^{\max }\right] .
\end{aligned}
$$

Consequently, the optimization problem (18) subject to (22), (23), and (24) can be solved as QP problem since these constraints are expressed as function of decision variables $\mathbf{w}_{1}$ and $\mathbf{F}_{c}^{r}$.

In the similar manner, by applying the following generalized torque-task relationship obtained from the contact consistent OSF

$$
\begin{array}{r}
\boldsymbol{\Gamma}=\widetilde{\mathbf{J}}_{1}^{T} \boldsymbol{\Lambda}_{1}\left(\ddot{\mathbf{x}}_{1}^{d}+\mathbf{w}_{1}\right)+\sum_{i=2}^{m}\left\{\left(\prod_{j=1}^{i-1} \widetilde{\mathbf{N}}_{j}^{T}\right) \widetilde{\mathbf{J}}_{i}^{T} \boldsymbol{\Lambda}_{i}\left(\ddot{\mathbf{x}}_{i}^{d}+\mathbf{w}_{i}\right)\right\} \\
+\mathbf{Y}_{c} \mathbf{F}_{c}^{r}+\boldsymbol{\Gamma}_{h},
\end{array}
$$

instead of (19), QP formulation of $l$-th priority task can be expressed as follows:

$$
\min _{\mathbf{w}_{l}, \mathbf{F}_{c}^{r}}\left\|\mathbf{w}_{l}\right\|^{2}
$$

subject to

$$
\begin{aligned}
& \boldsymbol{\Gamma}=\widetilde{\mathbf{J}}_{1}^{T} \boldsymbol{\Lambda}_{1}\left(\ddot{\mathbf{x}}_{1}^{d}+\mathbf{w}_{1}^{*}\right)+\sum_{i=2}^{l-1}\left\{\left(\prod_{j=1}^{i-1} \widetilde{\mathbf{N}}_{j}^{T}\right) \widetilde{\mathbf{J}}_{i}^{T} \boldsymbol{\Lambda}_{i}\left(\ddot{\mathbf{x}}_{i}^{d}+\mathbf{w}_{i}^{*}\right)\right\} \\
& +\prod_{j=1}^{l-1}\left\{\widetilde{\mathbf{N}}_{j}^{T} \widetilde{\mathbf{J}}_{l}^{T} \boldsymbol{\Lambda}_{l}\left(\ddot{\mathbf{x}}_{l}^{d}+\mathbf{w}_{l}\right)\right\}+\mathbf{Y}_{c} \mathbf{F}_{c}^{r}+\boldsymbol{\Gamma}_{h} \in\left[\boldsymbol{\Gamma}^{\min }, \boldsymbol{\Gamma}^{\max }\right] \\
& \ddot{\mathbf{x}}_{l}^{d}+\mathbf{w}_{l} \in\left[\ddot{\mathbf{x}}^{\min }, \ddot{\mathbf{x}}^{\max }\right], \text { and } \\
& \mathbf{K} \mathbf{F}_{c}=\mathbf{K} \overline{\mathbf{J}}_{c}^{T} \mathbf{S}^{T} \widetilde{\mathbf{J}}_{1}^{T} \boldsymbol{\Lambda}_{1}\left(\ddot{\mathbf{x}}_{1}^{d}+\mathbf{w}_{1}^{*}\right) \\
& \quad+\mathbf{K} \overline{\mathbf{J}}_{c}^{T} \sum_{i=2}^{l-1}\left\{\left(\prod_{j=1}^{i-1} \widetilde{\mathbf{N}}_{j}^{T}\right) \widetilde{\mathbf{J}}_{i}^{T} \boldsymbol{\Lambda}_{i}\left(\ddot{\mathbf{x}}_{i}^{d}+\mathbf{w}_{i}^{*}\right)\right\} \\
& \quad+\mathbf{K} \overline{\mathbf{J}}_{c}^{T} \prod_{j=1}^{l-1}\left\{\widetilde{\mathbf{N}}_{j}^{T} \widetilde{\mathbf{J}}_{l}^{T} \boldsymbol{\Lambda}_{l}\left(\ddot{\mathbf{x}}_{l}^{d}+\mathbf{w}_{i}\right)\right\} \\
& \quad+\mathbf{K} \overline{\mathbf{J}}_{c}^{T} \mathbf{S}^{T}\left(\mathbf{Y}_{c} \mathbf{F}_{c}^{r}+\mathbf{\Gamma}_{h}\right)+\mathbf{K} \mathbf{h}_{c} \in\left[\mathbf{f}_{c}^{\min }, \mathbf{f}_{c}^{\max }\right] .
\end{aligned}
$$

Note that the second term of the right-hand side of (28) and (29) has to be ignored when $l=2$.

With the proposed HQP formulation, the cascade QPs can be solved in order from $l=1$ to $m$. As a result, optimal slack variables $\mathbf{w}_{1}, \ldots, \mathbf{w}_{m}$ can be obtained from each corresponding QP, and $\mathbf{F}_{c}^{r}$ can be obtained from the last QP. Finally, control torque can be computed by submitting the optimal solutions $\mathbf{w}_{1}, \ldots, \mathbf{w}_{m}$ and $\mathbf{F}_{c}^{r}$ into (25).

Like the conventional HQP, strict task hierarchy is obtained by applying the optimal slack variables into equality constraint (25). In addition, the constraint (25) effects like the three constraints (3), (13), and (5) for the conventional HQP since the OSF is obtained based on those constraints while minimizing the joint acceleration energy. Therefore, the conventional HQP approach can produce the same optimal solution as the proposed method when the joint acceleration energy is minimized after solving QPs for the tasks [14].

\section{B. Discussions}

1) Contact wrench optimization: When a robot is in multi-contact, i.e., when the rank of $\mathbf{J}_{c}>6$, there is an infinite number of contact wrench solutions. Accordingly, an 
irregular and discontinuous solution can be given because the contact wrench is not optimized in the proposed method described in the previous subsection. Therefore, after obtaining the optimal slack variables, it is recommended to optimize the contact wrench. For this purpose, the following QP can be solved at the end of the proposed HQP for the contact wrench optimization: $\min _{\mathbf{F}_{c}^{r}}\left\|\mathbf{W}_{c} \mathbf{F}_{c}\right\|^{2}$ subject to (27), (28), and (29), where $\mathbf{W}_{c}$ is a positive constant diagonal matrix for weighting. Optimal $\mathbf{F}_{c}^{r}$ obtained from the above QP is then substituted into (25) when calculating the desired torque.

On the other hand, when a robot has a single plane contact, i.e., when the rank of $\mathbf{J}_{c}$ is 6 , the contact wrench has a unique solution. This is because 6 DoFs virtual joints describing floating base motion can be created by the 6 DoFs contact wrench. Therefore, the terms related to $\mathbf{F}_{c}^{r}$ can be removed from the developed HQP formulation and the contact wrench optimization is unnecessary. The HQP can be solved faster than when there is contact redundancy accordingly.

2) Optimization of force-level tasks: Explicit force-level tasks can be taken into account in the proposed HQP approach instead of the acceleration-level tasks. They can be optimized by applying the following equation as constraint instead of (25):

$\boldsymbol{\Gamma}=\widetilde{\mathbf{J}}_{1}^{T}\left(\mathbf{F}_{1}^{d}+\mathbf{w}_{1}\right)+\sum_{i=2}^{m}\left\{\left(\prod_{j=1}^{i-1} \widetilde{\mathbf{N}}_{j}^{T}\right) \widetilde{\mathbf{J}}_{i}^{T}\left(\mathbf{F}_{i}^{d}+\mathbf{w}_{i}\right)\right\}+\mathbf{Y}_{c} \mathbf{F}_{c}^{r}+\boldsymbol{\Gamma}_{h}$,

where $\mathbf{F}_{\bullet}^{d}$ is the desired force vector of $\bullet$-th priority task. It is applicable since the force and acceleration relationship at the operational space, i.e., $\mathbf{F}=\boldsymbol{\Lambda} \ddot{\mathbf{x}}$ [5], is satisfied in the OSF. For the sake of convenience, all tasks are expressed as force in (30), but whether to control acceleration or force can be selected for each task. For instance, when the first priority is an acceleration-level task and the second priority is a force-level task, the torque-task relationship constraint can be expressed as follows:

$$
\boldsymbol{\Gamma}=\widetilde{\mathbf{J}}_{1}^{T} \boldsymbol{\Lambda}\left(\ddot{\mathbf{x}}_{1}^{d}+\mathbf{w}_{1}\right)+\widetilde{\mathbf{N}}_{1}^{T} \widetilde{\mathbf{J}}_{2}^{T}\left(\mathbf{F}_{2}^{d}+\mathbf{w}_{2}\right)+\mathbf{Y}_{c} \mathbf{F}_{c}^{r}+\boldsymbol{\Gamma}_{h}
$$

The capability of the force-level tasks is an advantage of the proposed method compared to the conventional HQP-based methods that can only perform acceleration-level tasks.

3) Multi-threading: The parameters of the OSF require a large amount of computation. When both the OSF and HQP are calculated in a real-time control loop, the total computation time of the proposed method can be longer than that of the conventional HQP. Therefore, we compute OSF parameters by utilizing multi-threading of the computer through the method we previously proposed for QP-based method [18]. The multi-threading is a popular method for the modern robot for reducing computational burden in the real-time loop.

The OSF parameters contain the operational space dynamics parameters for all of the hierarchies $\left(\Lambda_{\bullet}, \widetilde{\mathbf{J}}_{\bullet}^{T}, \widetilde{\mathbf{N}}_{\bullet}^{T}\right)$, contact space dynamics parameters $\left(\mathbf{Y}_{c}, \overline{\mathbf{J}}_{c}^{T}, \boldsymbol{\mu}_{c}, \mathbf{p}_{c}\right)$, and the compensation torque $\boldsymbol{\Gamma}_{h}$. By calculating the OSF parameters in a non-real-time thread, only the HQP has to be solved

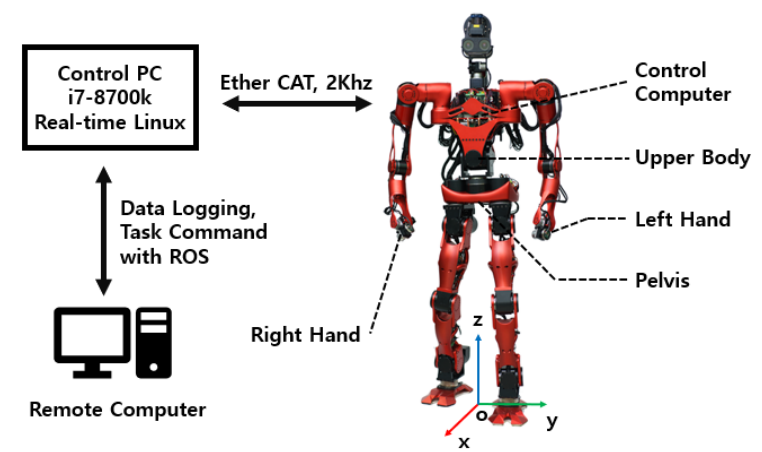

Fig. 1. The realtime control setup of the humanoid robot TOCABI.

TABLE I

CONFIGURATION OF HIERARCHICAL TASKS

\begin{tabular}{clcc}
\hline \hline Priority & Task & Motion DoFs & Contact DoFs \\
\hline 1 & T1-CoM pos $(x, y, z) \&$ Pelvis ori (roll, pitch , yaw) & 6 & 12 \\
2 & T2-Both hands pos $(x, y, z)$ \& ori (roll, pitch, yaw) & 12 & 12 \\
3 & T3-Upper-body ori (roll, pitch, yaw) & 3 & 12 \\
4 & T4-Joint damping & 33 & 12 \\
5 & T5-Contact wrench optimization & 0 & 12 \\
\hline \hline
\end{tabular}

for implementing the proposed method in a real-time thread. The real-time thread read the most recently calculated OSF parameters from the non-real-time thread and solves the HQP in each control loop. In this way, the computation of the OSF parameters can be separated from the real-time thread.

\section{EXPERIMENTAL VERIFICATION}

\section{A. Experimental setup}

To evaluate, the proposed HQP-based WBC method is implemented in a human-sized $(1.8 \mathrm{~m}$ height and $100 \mathrm{~kg}$ weight) 33 DoFs torque-controllable humanoid robot TOCABI shown in Fig. 1. The robot has 8 DoFs for each arm, 6 DoFs for each leg, 3 DoFs for the waist, and 2 DoFs for the neck. Each joint motor is torque-controlled with the current control-based servo drive which communicates with the real-time control computer (Xenomai-3.0 on Linux 4.14) through the EtherCAT interface. The proposed method is implemented in the computer with $3.7 \mathrm{GHz}$ octa-core processor and 16 GByte memory. QPs are solved by qpOASES library and Eigen linear algebra library. The sampling frequency of the real-time loop for whole-body control is set to $1 \mathrm{kHz}$ while the frequency of the communication is $2 \mathrm{kHz}$.

For the verification, five hierarchical tasks are assigned as shown in Table I with two plane contacts at feet. The first priority task (T1) is for controlling the position of the center of mass $(\mathrm{CoM})$ and orientation of the pelvis. The second priority task (T2) is to control the position and orientation of both hands. The third priority task (T3) is to control the orientation of upper-body, and the fourth priority task (T4) is for generating damping torque in all joints with $\boldsymbol{\Gamma}=-k_{v j} \dot{\mathbf{q}}$, where $k_{v j}$ is gain. In the last priority (T5), contact wrench is optimized to minimize contact moments and tangential forces with the optimization proposed in Sec. III-B.1. For this, the third and ninth diagonal component of $\mathbf{W}_{c}$ are set as zero and the others are set as one since the third and ninth component 


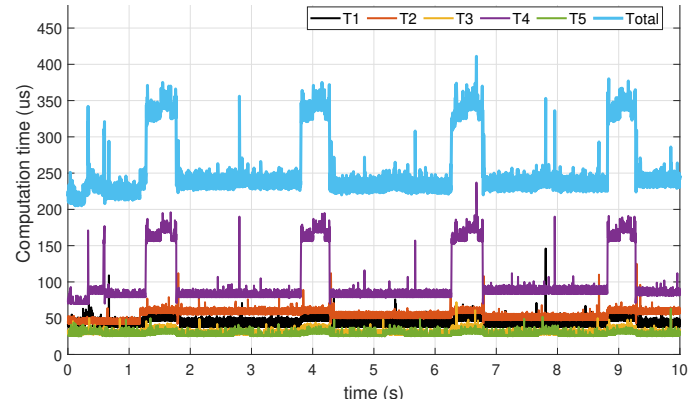

Fig. 2. Total HQP computation time and each $\mathrm{QP}$ computation time during robot experiment. Plot results contain QP formulating and solving time.

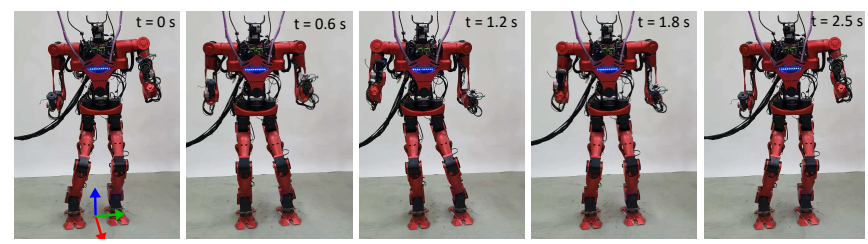

Fig. 3. Snapshots of controlled motion with the proposed method, which is repeated for 40 seconds (see the attached video for the full experiment.)

of $\mathbf{F}_{c}$ describes normal contact force. For the motion control, the desired accelerations $\left(\ddot{\mathrm{x}}^{d}\right)$ are determined to track desired position $\mathbf{x}^{d}$ and velocity $\dot{\mathbf{x}}^{d}$ with the following PD control scheme: $\ddot{\mathbf{x}}^{d}=k_{p}\left(\mathbf{x}^{d}-\mathbf{x}\right)+k_{v}\left(\dot{\mathbf{x}}^{d}-\dot{\mathbf{x}}\right)$, where $k_{p}, k_{v}$ are proportional and derivative gains, respectively. For all the tasks, joint torque limit (21) and contact wrench constraints (22) are set as inequality constraints.

\section{B. Results}

Solving time for each QP and the total HQP solving time during the experiment can be seen in Table II and Fig. 2. The solving time contains time for formulating and solving time of the QP problem. It can be seen that the calculation time increases in proportion to the task DoFs which related with the decision variable size. The computation time is not uniform during the experiment since the number of iteration for QP solving can be different in accordance with the QP problem. The total calculation is completed within 0.42 ms with the proposed HQP and the control frequency with $1 \mathrm{kHz}$ is successfully performed. There is time margin after solving the HQP for a given task, so it is possible to solve the HQP with more prioritized tasks or conduct higher control frequency. The computation of OSF parameters in the non-real-time thread is completed at $0.41 \mathrm{~ms}$ on average. Therefore, the OSF parameters can also be updated in $1 \mathrm{kHz}$ frequency in most cases.

Whole-body motion controlled by the proposed HQP is shown in Fig. 3, and the corresponding trajectories and control results of the tasks $\mathrm{T} 1, \mathrm{~T} 2$, and $\mathrm{T} 3$ are shown in Fig. 4. Repetitive motion with $2.5 \mathrm{~s}$ period is controlled during $40 \mathrm{~s}$, and the partial result for $5 \mathrm{~s}$ are described in Fig. 4. According to the given trajectory, the CoM moves in $y$-axis, both hands move up and down in $z$-axis, and yaw angle of the upper-body rotates repetitively. Average of 2norm of the control error during $40 \mathrm{~s}$ experiment of the
TABLE II

HQP SOLVING TIME OF THE PROPOSED METHOD

\begin{tabular}{ccccccc}
\hline \hline solving time & $\mathrm{T} 1$ & $\mathrm{~T} 2$ & $\mathrm{~T} 3$ & $\mathrm{~T} 4$ & $\mathrm{~T} 5$ & Total \\
\hline min. (ms) & 0.037 & 0.041 & 0.027 & 0.070 & 0.025 & $\mathbf{0 . 2 0 5}$ \\
max. (ms) & 0.146 & 0.129 & 0.072 & 0.237 & 0.063 & $\mathbf{0 . 4 1 7}$ \\
average $(\mathrm{ms})$ & 0.044 & 0.055 & 0.030 & 0.097 & 0.027 & $\mathbf{0 . 2 5 3}$ \\
\hline \hline
\end{tabular}

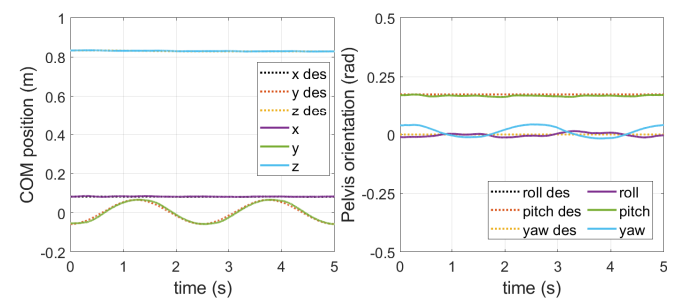

(a) T1: CoM and pelvis orientation

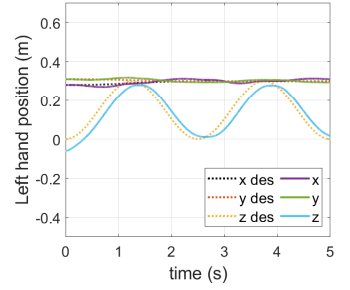

(b) T2: hands position

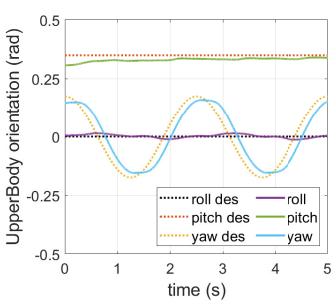

(c) T3: upper-body orientation
Fig. 4. Tracking responses of the assigned tasks (responses of the right hand in $\mathrm{T} 2$ (b) are omitted due to the similarity to those of the left hand.)

TABLE III

AVERAGE HQP SOLVING TIME COMPARISON IN SIMULATIONS

\begin{tabular}{ccccccc}
\hline \hline solving time & T1 & T2 & T3 & T4 & T5 & Total \\
\hline conventional $(\mathrm{ms})$ & 1.416 & 1.764 & 1.380 & 3.176 & 1.527 & 9.263 \\
proposed $(\mathrm{ms})$ & 0.059 & 0.068 & 0.041 & 0.099 & 0.038 & $\mathbf{0 . 3 0 3}$ \\
\hline \hline
\end{tabular}

CoM is $0.0055 \mathrm{~m}$, pelvis orientation is $0.0237 \mathrm{rad}$, both hands position is $0.0350 \mathrm{~m}$, and upper body orientation $0.0459 \mathrm{rad}$. Through the trajectory tracking control results, one can notice that the proposed HQP effectively controls the multiple tasks as desired while keeping stable contacts.

\section{Comparative Analyses of Computation Time}

In this section, for fair comparison of the proposed method with the conventional HQP introduced in Sec. II, we perform simulations of WBC with the same tasks in Table I. The simulation is implemented in $\mathrm{MuJoCo}$ with a $4.0 \mathrm{GHz}$ quadcore processor (i7-6700K) and 16 GByte memory.

\section{A. Result of Computation Time Comparisons}

Average computation time of both methods in the simulations are shown in Table III. The proposed method solves the HQP averagely in $0.3 \mathrm{~ms}$ and the conventional HQP solves the same hierarchical tasks averagely in $9.26 \mathrm{~ms}$. Due to the reduced decision variable size, the proposed method can solve the HQP approximately 30.5 times faster. Both the size of the decision variable increases in accordance with the task DoFs so all the computation time of each QP is proportional to the decision variable size. 
Meanwhile, unlike the conventional method, the proposed method requests to calculate the OSF parameters in addition to computing QPs. Nevertheless, the proposed method can compute faster even if the multi-threading is not performed for the OSF parameters calculation, i.e., when the OSF parameters are computed in the real-time thread. The OSF parameters require $0.64 \mathrm{~ms}$ computation time on average so $0.94 \mathrm{~ms}$ on average is required to solve the HQP, which is 9.8 times faster than the conventional method.

\section{B. Comparison with Other Advanced HQP Methods}

Computation cost of the conventional HQP in Sec. II can be reduced by adopting algorithms proposed in other studies. Thus, we present additional discussions to compare with the typical methods for fast HQP solving [13]-[15].

The method in [14] reduces the computation cost by decreasing the size of the decision variable. They reduce the variable size to $k+6$, which is the same as the variable size of T4 for the proposed method - T4 is the worst task in terms of computation time. In the proposed method, tasks with DoFs as much as the number of joints $(k)$ are generally assigned one or less. Therefore, our method will require less computation time for solving HQP in most cases. Moreover, the difference in the amount of computation between the two methods will become larger as the number of task hierarchy increases. It can be seen by the experimental results in the literature that our method is faster. The method in [14] solves the HQP approximately 4 to 5 times faster than the method in Sec. II, while our method solves 30.5 times faster.

The authors in [13] enhances computation speed by decreasing both variable size and number of constraints by using methods in [11], [12]. The simulation result with a 25 DoFs humanoid in the study shows that they solve the HQP about 1.6 times faster than when the decision variable size $n+c$ is the same as the method in Sec. II. It takes up to $3 \mathrm{~ms}$ to solve HQP for three hierarchical tasks. Even considering the lower computing power of their computer, the computation time is much longer than our method.

By comparing the decision variable size and the results of experiments and simulations in the literature [13], [14], we can infer that the proposed method is faster. However, we cannot conclude how much faster our method is compared to the other methods since the computer, robot, QP solver, constraints, task configuration, etc. are all different. Therefore, in order to make a more precise comparison, various experiments have to be performed in future studies with the same robot and computer system.

Unlike the above studies, the study in [15] proposes the HQP solving method with analytic approach. Computation time of the inverse kinematics-based WBC problem with many hierarchies drastically decreased by this algorithm. To the best of our knowledge, the method has not yet been implemented for inverse dynamics control in real humanoid robot hardware although it can be applied for the inverse dynamics problem. Therefore, to improve our method, it can be further extended for our proposed method in the future.

\section{CONCLUSION}

In this study, we propose the OSF-based HQP for wholebody control of floating base robots. By adopting the contact consistent OSF, the computation cost of the HQP approach is decreased with reduced decision variable size. Consequently, the HQP can be solved faster than the conventional methods. The performance is evaluated by real robot experiment with 33 DoFs humanoid robot with five hierarchical tasks. The HQP can be solved within $0.42 \mathrm{~ms}$, and motions are successfully controlled with $1 \mathrm{kHz}$ control frequency accordingly. In addition, it is notable that the decision variable size of the proposed HQP is regardless of the robot joint numbers so the proposed method can be more effectively utilized for high-DoFs robots than the other HQP methods.

\section{REFERENCES}

[1] C. Collette, A. Micaelli, C. Andriot, and P. Lemerle, "Robust balance optimization control of humanoid robots with multiple non coplanar grasps and frictional contacts," in Proc. 2008 IEEE Int. Conf. Robot. and Automat. (ICRA), 2008, pp. 3187-3193.

[2] S. Kuindersma, F. Permenter, and R. Tedrake, "An efficiently solvable quadratic program for stabilizing dynamic locomotion," in Proc. 2014 IEEE Int. Conf. Robot. and Automat. (ICRA), 2014, pp. 2589-2594.

[3] S. Feng, E. Whitman, X. Xinjilefu, and C. G. Atkeson, "Optimizationbased full body control for the darpa robotics challenge," J. Field Robot., vol. 32, no. 2, pp. 293-312, 2015.

[4] G. Mesesan et al., "Dynamic walking on compliant and uneven terrain using dcm and passivity-based whole-body control," in 2019 IEEERAS Int. Conf. Humanoid Robots (Humanoids), 2019, pp. 25-32.

[5] J. Park and O. Khatib, "Contact consistent control framework for humanoid robots," in Proc. 2006 IEEE Int. Conf. Robot. and Automat. (ICRA), 2006, pp. 1963-1969.

[6] Y. Lee, S. Hwang, and J. Park, "Balancing of humanoid robot using contact force/moment control by task-oriented whole body control framework," Auton. Robots, vol. 40, no. 3, pp. 457-472, 2016.

[7] D. Kim et al., "Stabilizing series-elastic point-foot bipeds using wholebody operational space control," IEEE Trans. Robot., vol. 32, no. 6, pp. 1362-1379, 2016.

[8] Y. Lee, H. Lee, J. Lee, and J. Park, "Toward reactive walking: Control of biped robots exploiting an event-based fsm," IEEE Transactions on Robotics, pp. 1-16, 2021, (in press).

[9] H. Han and J. Park, "Robot control near singularity and joint limit using a continuous task transition algorithm," Int. J. Adv. Robot. Syst., vol. 10, no. 10, p. 346, 2013.

[10] M. De Lasa and A. Hertzmann, "Prioritized optimization for taskspace control," in 2009 IEEE/RSJ Int. Conf. Intelligent Robots and Systems (IROS), 2009, pp. 5755-5762.

[11] M. De Lasa, I. Mordatch, and A. Hertzmann, "Feature-based locomotion controllers," ACM Trans. Graph., vol. 29, no. 4, pp. 1-10, 2010.

[12] O. Kanoun, F. Lamiraux, and P.-B. Wieber, "Kinematic control of redundant manipulators: Generalizing the task-priority framework to inequality task," IEEE Trans. Robot., vol. 27, no. 4, pp. 785-792, 2011.

[13] A. Herzog et al., "Momentum control with hierarchical inverse dynamics on a torque-controlled humanoid," Auton. Robots, vol. 40, no. 3, pp. 473-491, 2016.

[14] N. Mansard, "A dedicated solver for fast operational-space inverse dynamics," in Proc. 2012 IEEE Int. Conf. Robot. and Automat. (ICRA), 2012, pp. 4943-4949.

[15] A. Escande, N. Mansard, and P.-B. Wieber, "Hierarchical quadratic programming: Fast online humanoid-robot motion generation," Int. J. Robot. Res., vol. 33, no. 7, pp. 1006-1028, 2014.

[16] J. Park, Control strategies for robots in contact. Citeseer, 2006.

[17] S. Caron, Q.-C. Pham, and Y. Nakamura, "Stability of surface contacts for humanoid robots: Closed-form formulae of the contact wrench cone for rectangular support areas," in 2015 IEEE Int. Conf. Robots. and Automat. (ICRA), 2015, pp. 5107-5112.

[18] Y. Lee et al., "A whole-body control framework based on the operational space formulation under inequality constraints via task-oriented optimization," IEEE Access, vol. 9, pp. 39 813-39 826, 2021. 